\title{
Imaging Modalities in Acute Ischemic Stroke
}

\author{
Kunal Bhatia ${ }^{1}$, Christopher R. Newey ${ }^{1}$, Naresh Karthikeyan ${ }^{1}$, Premkumar Nattanmai ${ }^{1}$ \\ ${ }^{1}$ Department of Neurology, University of Missouri Health Care, Columbia, MO
}

Correspondence: Christopher Newey (neweyc@ health.missouri.edu)

Received February 13, 2017; accepted March 28, 2017

As "time is brain", acute ischemic stroke is considered a medical emergency. With the introduction of thrombolytic therapy and availability of modern neuroimaging modalities, timely diagnosis of an ischemic lesion, exclusion of intracerebral hemorrhage, assessing the degree of brain injury, and evaluation of cerebral vasculature is necessary in acute stroke management. Various neuroimaging techniques have been used in selecting patients and guiding reperfusion therapies in patients with acute ischemic stroke during the initial hours of symptom onset. In this review we will highlight the importance of available imaging modalities used to assess patients with acute ischemic stroke.

Keywords: acute ischemic stroke, magnetic resonance imaging (MRI), computed tomography (CT)

\section{Background}

Stroke is the fifth leading cause of death accounting for approximately one in every 20 deaths in the United States (1). It is also a major cause of severe long-term disability (2). Stroke is classically characterized as an acute neurological deficit attributed to a vascular cause (3). Up to $87 \%$ are ischemic with $10 \%$ being intracerebral hemorrhage (ICH) and $3 \%$ being subarachnoid hemorrhage (SAH) strokes (2). The aim of neuroimaging in acute stroke is to obtain rapid information on tissue and vessel status to aid acute stroke intervention.

With the introduction of intravenous thrombolytic therapy and availability of modern neuroimaging modalities, timely diagnosis of an ischemic lesion and determining its vascular location and extent has led to efficient management of acute ischemic stroke and improved stroke outcomes (4-8).

The standard imaging modality used in the initial diagnosis of stroke is a non-contrast computed tomography (CT) scan of the head. This modality helps determine a hemorrhagic versus ischemic stroke (9). Magnetic resonance imaging (MRI) may eventually be substituted for $\mathrm{CT}$ as these become more readily available. MRIs can rapidly detect early ischemic lesions and the "ischemic penumbra" (10). One of the diagnostic advances with MRI is the diffusionweighted image (DWI) acquisition and apparent diffusion co-efficient (ADC) which allows early detection of an infarcted lesion (i.e., ischemic core) within minutes of a stroke (9).

In this review, we will highlight the various neuroimaging modalities currently available for diagnosing acute ischemic stroke and their utility in selecting patients for early reperfusion therapies, which has become a cornerstone of acute ischemic stroke intervention. 


\section{Ischemic Penumbra}

Acute ischemic stroke is caused by an abrupt cessation of blood supply to the brain causing a decrease in cerebral blood flow (CBF). There exists a compensatory auto-regulatory mechanism in the brain parenchyma that maintains a constant cerebral blood flow during the initial periods of hypoperfusion (11). This autoregulation is via recruitment of collaterals and dilatation of smaller arterioles (11). This allows for maximal oxygen extraction by the hypoperfused tissues to maintain tissue oxygenation and cellular viability (11). However, when the CBF falls below a threshold of 20 to $23 \mathrm{~mL} / 100 \mathrm{~g}$ tissue/min, neuronal function becomes impaired (11-14). Further drop in the CBF beyond this threshold leads to decrease in the cerebral perfusion pressure as the collaterals fail to provide tissue oxygenation, resulting in metabolic injury and tissue death (1114). The membrane channels ultimately fail causing a net shift of water into the cells resulting in cytotoxic edema and irreversible damage to the neuronal cells (11-14). This zone of irreversible cell injury is referred to as the "ischemic core". It is surrounded by a zone of tissue at risk of infarction referred to as the "ischemic penumbra" (14-16). The penumbra is potentially salvageable and is the target of various reperfusion strategies in the management of acute ischemic stroke.

With the development of functional imaging modalities, such as CT perfusion (CTP) and DWI/perfusion weighted MRI, salvageable tissue can be identified for timely reperfusion therapy (17).

\section{Computed Tomography (CT)}

Due to its widespread immediate availability, CT remains the most common and the first line imaging modality used in acute ischemic stroke (18). It is used to rule out hemorrhagic strokes (e.g., intracerebral hemorrhage $(\mathrm{ICH})$ ). By excluding $\mathrm{ICH}$, patients presenting within the therapeutic window ( 3 to 4.5 hours from symptom onset) may be eligible for intravenous tissue plasminogen activator (tPA) (19).

CT may demonstrate subtle early signs of ischemia. An early finding on CT indicating cerebral ischemia is loss of gray-white matter differentiation (Figure 1), which occurs from the increasing water concentration from ion pump failure (i.e., cytotoxic edema) $(20,21)$. It may be detected in the region of basal ganglia (i.e., lenticular obscuration) or insular cortex (i.e., insular ribbon sign) $(22,23)$. However, the inter-rater agreement in recognizing these CT findings is variable (24-29). These findings are also time dependent. They appear in $\leq 67 \%$ in patients imaged within 3 hours but increase to $82 \%$ at 6 hours (24-29). To improve the detection rates and inter-rater reliability, the Alberta stroke program early CT score (ASPECTS) was developed to assess early ischemic changes ( 3 hours from stroke onset) in patients with acute ischemic stroke of the anterior circulation $(30,31)$. It is a simple rating scale which divides the affected middle cerebral artery into ten segments: internal capsule, caudate nucleus, lentiform nucleus, insula, and six segments for cortical areas. One point is deducted for each area showing early ischemic changes. A score of 10 reflects a normal CT scan. A score of 0 indicates diffuse ischemic involvement throughout the complete middle cerebral artery (MCA) territory (30).

CT head may also show evidence of a thrombus within an artery, seen as an increased density in the transverse M1 segment (i.e., hyperdense MCA sign) (Figure 2) or in cross-section within the Sylvian fissure or basilar artery (i.e., dot sign) (32). This indicates vessel occlusion with a high specificity but moderate sensitivity of $30-40 \%$ (33). The appearance of this early sign of 
acute ischemic stroke may be associated with worse prognosis and an increased risk of thrombolysis-associated hemorrhage (34). It is a predictor of early neurological deterioration with a positive predictive value of $91 \%(35,36)$.

Ischemic strokes with hemorrhagic transformation are classified as either hemorrhagic infarction $(\mathrm{HI})$ or parenchymal hematoma $(\mathrm{PH})$. The hemorrhagic transformed strokes are then classified as HI1 (small petechiae), HI2 (more confluent petechiae), PH1 (<30\% of infarcted area with some mild space-occupying effect), and PH2 (> 30\% of the infarcted area with significant space-occupying effect) (37).

\section{Disadvantages:}

Although CT helps in excluding ICH, it cannot reliably differentiate between irreversibly damaged brain tissue (i.e., infarct core) and viable brain tissue (i.e., ischemic penumbra). Thus, it may be limited in selecting patients for various reperfusion therapies with an indeterminate time of symptom onset. It is also relatively insensitive in detecting acute and/or small cortical or subcortical infarctions, especially in the posterior fossa (38).
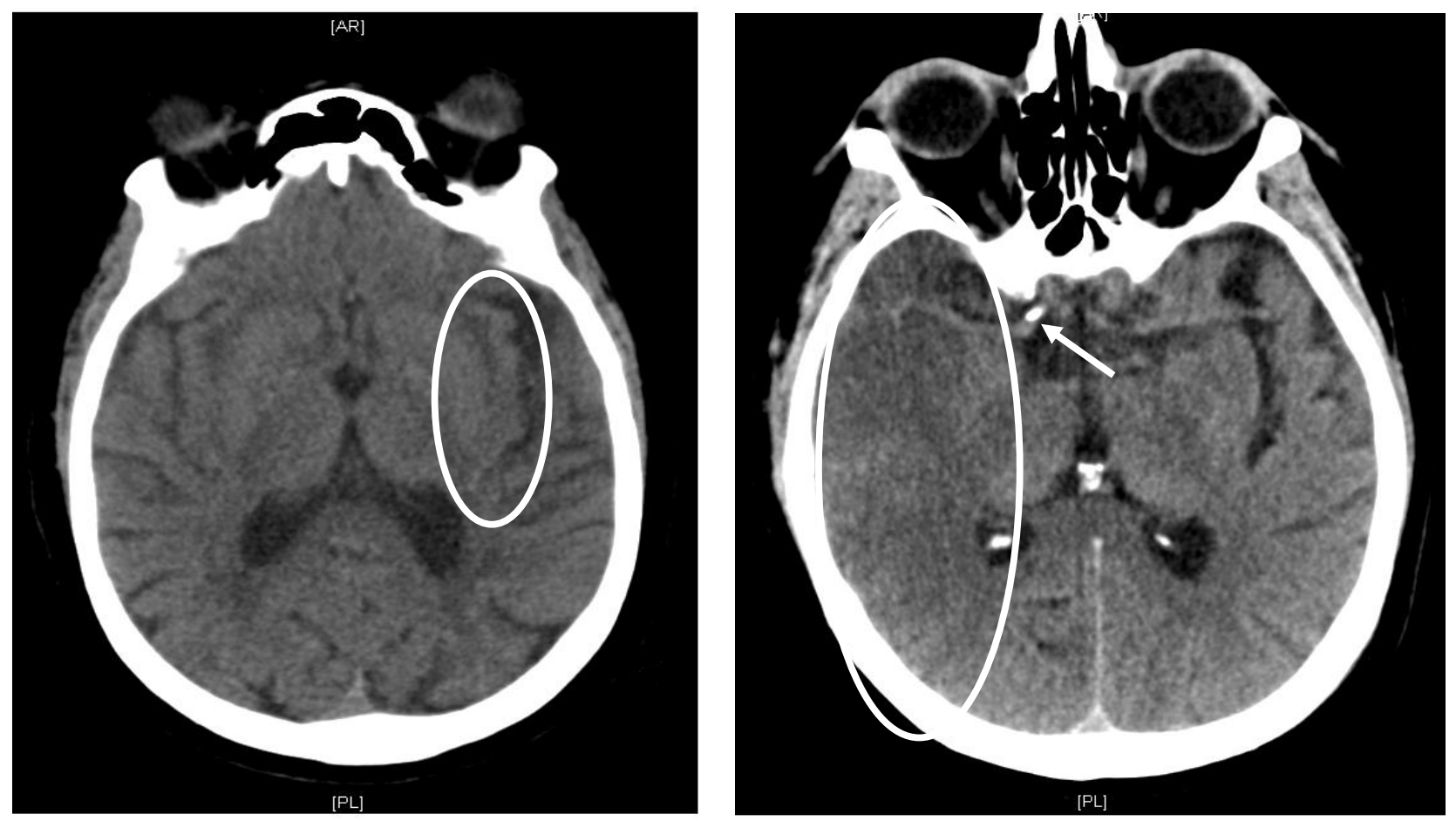

Figure 1 (left). Axial computed tomography. Loss of gray-white matter differentiation in the left insular region is seen on the left (circle). Figure 2 (right). Axial computed tomography. Evolving stroke is seen in the right middle cerebral artery (MCA) territory (circle). Hyperdense MCA sign is seen on the right MCA (arrow). 


\section{CT Angiography (CTA) and CT Perfusion (CTP) Imaging}

An important component of CT imaging protocol in the treatment of acute ischemic stroke is CT angiography (CTA), and at some centers CT perfusion (CTP) techniques. By allowing imaging of the intracranial vasculature, it can help identify the exact location and extent of vascular occlusion.

\section{$\underline{\text { CT Angiography (CTA) }}$}

CTA is a relatively rapid technique making it ideal in acute stroke management. It is a thinsection volumetric spiral (i.e., helical) technique following a time-optimized bolus of iodinated contrast medium to enhance visualization of the extra- and intra-cerebral circulations (39). The availability of new multidetector row CT technology and fast speed of acquisition of reformatted angiographic images has allowed visualization with high spatial resolution (39). It is useful in visualization of vessels from the aortic arch through the Circle of Willis within seconds (40). The sensitivity and specificity of CTA for the detection of intracranial occlusions is high ranging 92$100 \%$ and $82-100 \%$, respectively (41-44) (Figure 3). CTA is also sensitive and specific for imaging the extra-cranial vasculature as compared to carotid ultrasound in differentiating carotid occlusion from a very high grade stenosis (i.e., string sign) (45).

\section{Disadvantages:}

CTA requires the use of intravenous iodinated contrast medium, thus limiting its use in patients with contrast allergies and abnormal renal function.

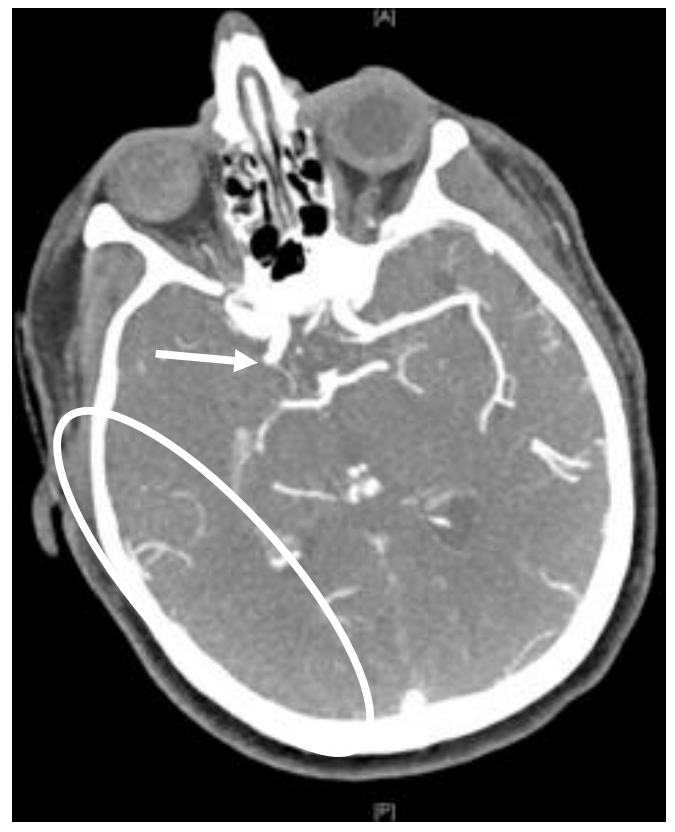

Figure 3. Axial computed tomography angiography (CTA). Occlusion of the right terminal internal carotid artery/proximal middle cerebral artery (MCA) is shown (arrow). Note the relative paucity of contrast filling vessels in the right MCA territory (circle) compared the left. This may indicate poor collateral circulation. 


\section{CT Perfusion (CTP)}

CTP is a functional brain imaging modality which requires the administration of an intravenous bolus of an iodinated contrast agent. This technique allows perfusion imaging of the brain parenchyma and is useful in differentiating infarct from ischemic penumbra (46). CTP can reliably predict and identify ischemic penumbra and the irreversible ischemic core in patients with acute ischemic stroke within a few minutes on admission with a sensitivity and specificity of $>90 \%$ (47). This is achieved by measuring the following parameters: cerebral blood flow (CBF), cerebral blood volume (CBV), mean transit time (MTT), and time to peak (TTP) (Table $1)$.

Table 1. CT/MRI Perfusion Imaging Parameters in Acute Ischemic Infarct

\begin{tabular}{|l|l|l|}
\hline & Ischemic penumbra & Infarct core \\
\hline MTT and TTP & Increased & Increased (threshold of 145\%) \\
\hline CBV & Normal to increased & Decreased \\
\hline CBF & Decreased $(<50 \%$ reduction) & Decreased $(>60 \%$ reduction) \\
\hline
\end{tabular}

MTT = mean transit time; TTP = time to peak. Cerebral blood volume (CBV) is the total volume of blood in a given unit of brain volume (normal $=4-5 \mathrm{~mL} / 100 \mathrm{~g}$ ). Cerebral blood flow $(\mathrm{CBF})$ is the volume of blood moving through a given unit of brain volume per unit time (normal range in grey matter $=50-60 \mathrm{~mL} / 100 \mathrm{~g} / \mathrm{min}$ ). Mean transit time (MTT) is the time difference between the arterial inflow and venous outflow (normal $=4$ secs in grey matter). Time to Peak Enhancement (TTP) is the time from the beginning of contrast material injection to the maximum concentration of contrast material within a region of interest (ROI). The relationship between CBF and CBV is expressed by the equation $\mathrm{CBF}=\mathrm{CBV} / \mathrm{MTT}$.

$\mathrm{CBF}$ is defined as the volume of blood flowing per $100 \mathrm{~g}$ of brain mass per minute $(48,49)$. It is expressed as $\mathrm{CBF}=\mathrm{CBV} / \mathrm{MTT}(48,49)$. Prolonged MTT together with $\mathrm{CBF}-\mathrm{CBV}$ mismatch is the most sensitive measure in detecting the tissue at risk of infarction. A decrease in total CBV is the most specific indicator of the ischemic core $(50,51)$. Typically, in patients with acute ischemic stroke, $\mathrm{CBF}$ and $\mathrm{CBV}$ are low in the infarct core; whereas a decrease in $\mathrm{CBF}$ with a normal or increased CBV along with a prolonged MTT is seen in the ischemic penumbra (52, 53) (Figure 4).

The ability of CTP to identify the penumbra makes it useful in treatment decisions in patients with indeterminate times of stroke onset or in cases of wake-up strokes (54). These patients may benefit from various reperfusion strategies. Figure 5 is an example of a right MCA occlusion seen on digital subtraction angiography (A) with recanalization of the superior division of the MCA (B). Any patient with a stroke (i.e., an acute neurological change within the past 24 hours) should be evaluated at a stroke center. At most stroke centers, a multimodal CT stroke protocol including CT head, CTP, and CTA is used. CTP imaging has the advantage of being performed during the same setting as CTA.

\section{Disadvantages:}

CTP requires repeated scanning of the same portion of the brain parenchyma until the contrast agent passes through the entire cerebral vasculature and produces a greater amount of radiation 
exposure. It also requires a multi-detector CT and a trained technician to set up the special software package and equipment. This limits its widespread availability. Use of a $2^{\text {nd }}$ bolus of iodinated contrast agent (after CTA) can predispose patients with renal insufficiency to contrastinduced nephropathy.

CT, CTA, and CTP all have the disadvantage of radiation exposure.

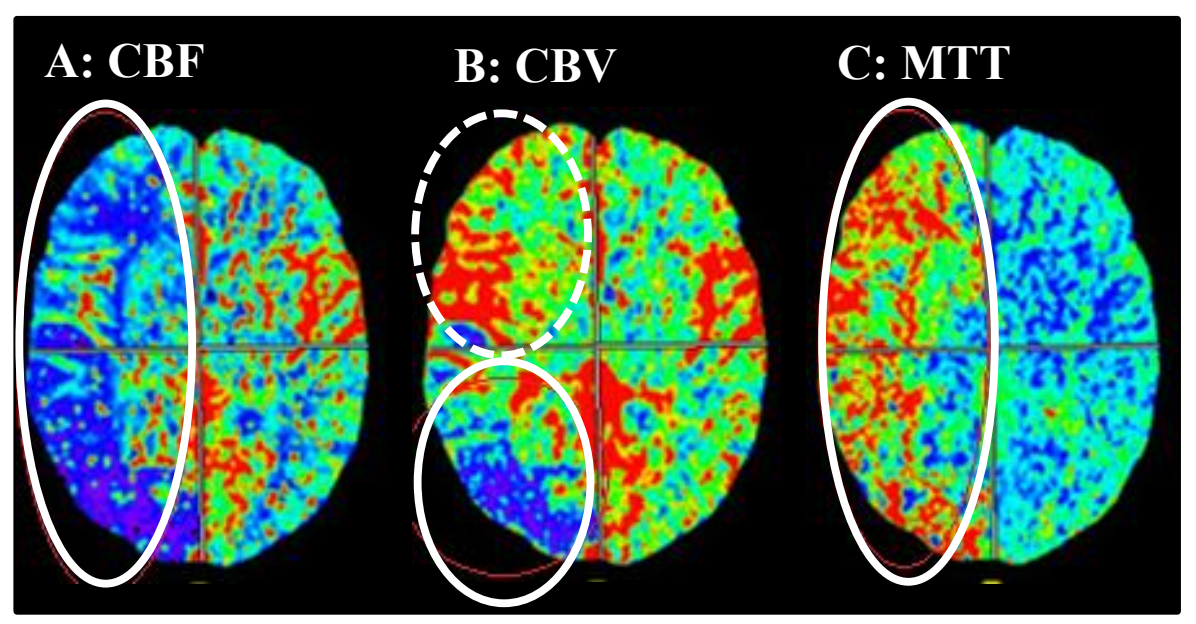

Figure 4. Computed tomography perfusion. A) Cerebral blood flow (CBF) showing decreased (circle) area of flow. B) Cerebral blood volume (CBV) showing area of decreased volume (solid circle) indicating core infarct with surrounding area of normal/increased CBV consistent with area of penumbra (dotted circle). C) Mean transit time (MTT) showing area of increased transit.

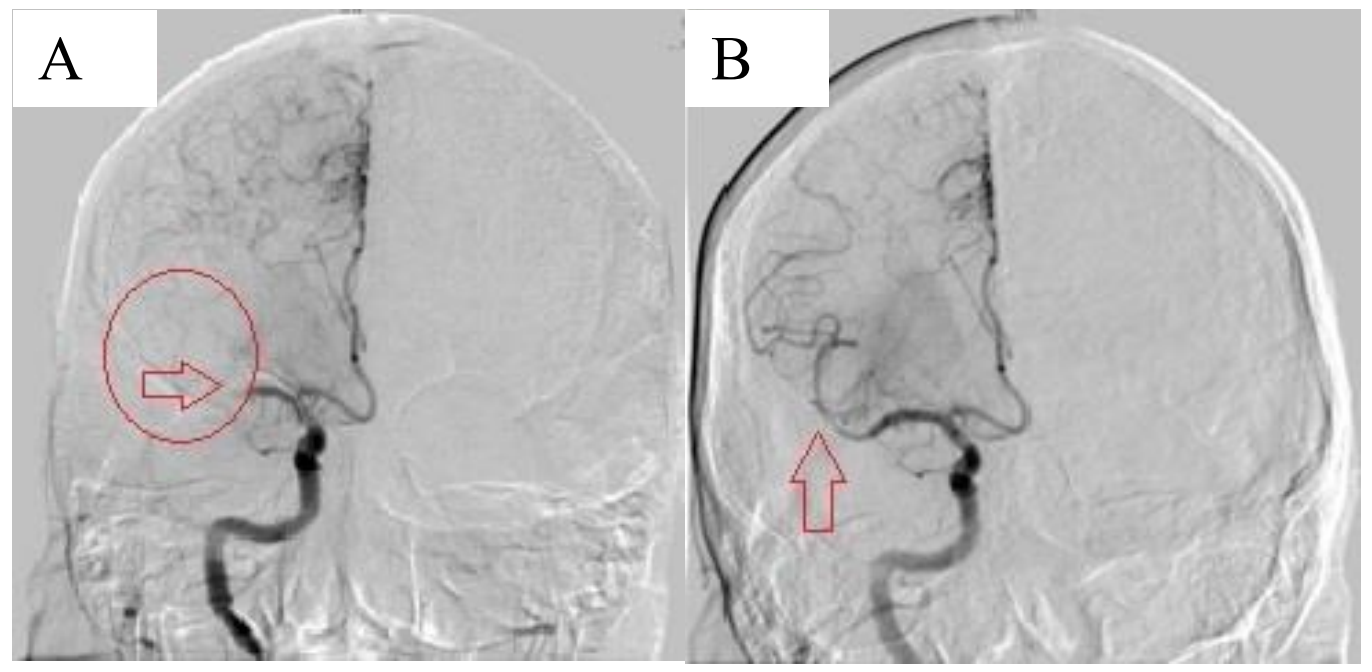

Figure 5. Cerebral angiography. A) Acute occlusion of the right middle cerebral artery (MCA) is seen (arrow) with paucity of distal flow (circle). B) Restoration of flow in the superior division (arrow) after endovascular thrombectomy using stent retriever. 


\section{Magnetic Resonance Imaging (MRI)}

Brain magnetic resonance imaging (MRI) imaging protocols for acute ischemic stroke include T1- and T2-weighted sequences, fluid attenuated inversion recovery (FLAIR) sequence, perfusion-weighted imaging (PWI), and DWI with corresponding ADC maps (46). These sequences can confirm the diagnosis of ischemia and guide the selection of patients for IV-tPA and other reperfusion strategies (46).

Conventional brain MRI sequences are not sensitive for detecting cytotoxic edema in the acute phase, whereas T1 and T2 weighted images can detect vasogenic edema and can help in differentiating acute versus subacute stroke (55-57). T2-weighted and FLAIR images can detect acute ischemic tissue within 3-8 hours after stroke onset, seen as hyperintense signals, with loss of grey-white matter differentiation and sulcal effacement similar to that seen with CT (55-57). FLAIR sequences are also useful in detecting subarachnoid hemorrhages (seen as bright signal) by suppressing the CSF signal (dark), as compared to T1 and T2 weighted images.

The gradient recalled echo (GRE) or susceptibility weighted image (SWI) sequences of MRI are more sensitive for detection of blood products and chronic hemorrhages, particularly chronic microbleeds, due to the paramagnetic effect of the hemosiderin. (58). These are seen as "blooming" effects and black on GRE images. They are used to assess the burden of underlying chronic vascular disease in stroke patients (59).

In comparison to T2 weighted and FLAIR sequences, DWI is the most sensitive (88-90\%) and specific (95-100\%) imaging technique for detecting acute ischemic change (60-64). Changes can be seen within a few minutes of symptom onset (60-64). Acquisition is based on the ability of diffusion weighted MRI to quantify motion of water molecules accumulated intracellularly in the injured neurons relative to extracellular space. This allows identification of ischemic cells on the DWI images which appear bright $(46,65)$. This should always be correlated with the ADC maps. Therefore, an increased signal on DWI (hyperintense) together with a decreased signal on ADC map represents an area of brain infarction (Figure 6). This sequence complement is known as restricted diffusion. DWI and ADC maps together with T2-weighted images and FLAIR sequences can be used to distinguish acute lesions from subacute or older infarcts $(66,67)$. In general, lesions appearing hypointense on ADC map are usually $<7-10$ days old whereas lesions seen as isointense or hyperintense are more likely $>7-10$ days old $(66,67)$. Lesions on DWI can also be used to identify stroke etiology. For example, isolated lenticulostriate lesion points to lacunar infarct whereas multiple lesions in different vascular territories suggest a cardioembolic source. Large artery atherosclerosis can manifest as scattered lesions within one vascular territory (6).

\section{Perfusion-Weighted Imaging (PWI)}

The combination of DWI and PWI is commonly used in clinical practice to evaluate the extent of irreversible tissue damage and to identify ischemic penumbra $(68,69)$. DWI along with ADC can identify tissue with permanent damage, whereas areas without signal change on DWI-ADC, but abnormal signal on PWI, represent salvageable tissue at risk $(68,69)$. This area is the ischemic penumbra. A mismatch is considered significant if the penumbra is at least $20 \%$ of the ischemic core volume (70). The mismatch pattern is found in about $70 \%$ of all patients with anterior circulation stroke within 6 hours of onset, and may represent an indication for reperfusion treatment (69). 


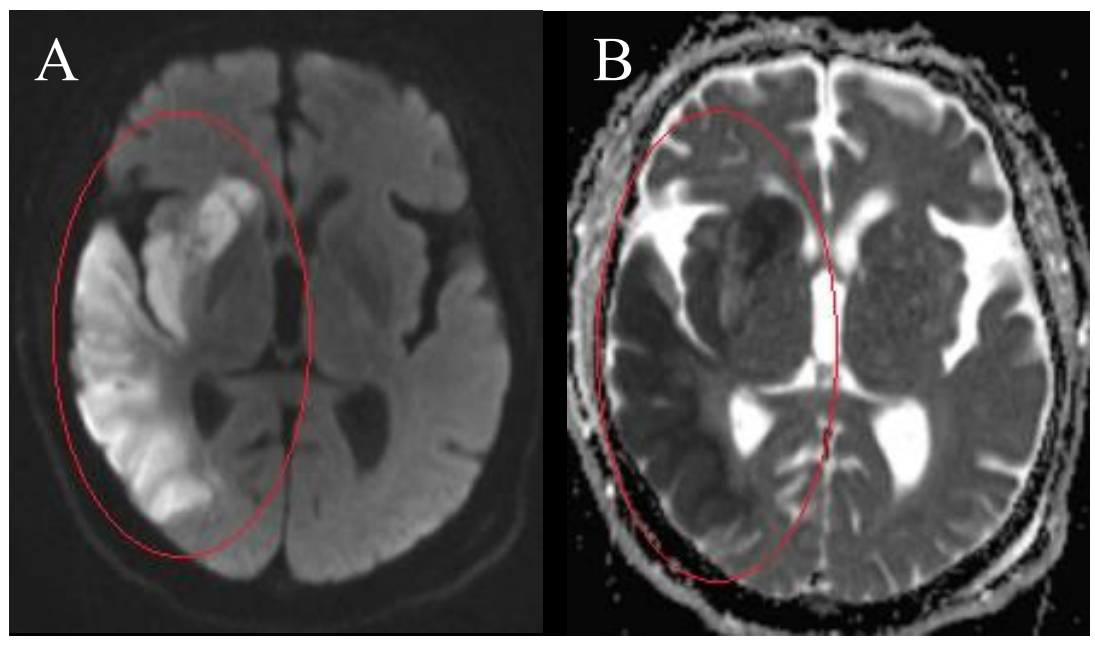

Figure 6. Magnetic resonance imaging (MRI) diffusion weighted imaging (DWI) and apparent diffusion coefficient (ADC). A) DWI showing area of hyperintensity in the right middle cerebral artery (MCA) distribution (circle). B) ADC showing hypointensity in the same MCA distribution (circle). The combination of DWI hyperintensity and $\mathrm{ADC}$ hypointensity is known as restricted diffusion.

Magnetic resonance perfusion (MRP) parameters are similar to the ones seen on CTP (Table 1). However, these are more difficult to quantify as compared to CTP. Time to peak (TTP) and MTT are preferred for identifying hypoperfused tissue and cerebral brain perfusion; MTT and CBF denote ischemic penumbra and can predict the final infarct volume in absence of reperfusion (70, 71). In contrast to the CTP, there is availability of contrast-induced PWI technique which incorporates the use of gadolinium based contrast through the cerebral vasculature and measures various perfusion parameters but with lesser accuracy as compared to CTP.

$\mathrm{MRP}$, as compared to CTP, has the advantage of imaging the entire brain parenchyma. MRP is more precise in predicting the tissue at risk and hence the final infarct volume and does not carry the hazard of radiation exposure or exposure to contrast agents as with CT perfusion protocol.

\section{MR Angiography (MRA)}

Magnetic resonance angiography (MRA) is a noninvasive technique used to evaluate extracranial and intracranial vessels and to identify the site of vascular occlusion or stenosis in patients with ischemic stroke (72). Several MRA techniques have been developed to image intra- and extracerebral vessels. They include 2-dimensional time-of-flight (TOF), 3-dimensional TOF, multiple overlapping thin-slab acquisition (MOTSA), and contrast enhanced MRA (CE-MRA) (73).

Intracranial MRA has a sensitivity of $60-85 \%$ for stenosis and $80-90 \%$ for occlusions compared with CTA or digital subtraction angiography (DSA) (74). As compared to CTA, it can avoid the use of contrasted agents. In some cases, it overestimates the degree of vessel stenosis and motion artifacts as it is based on time of flight of blood flow.

Similarly, CE-MRA is more accurate with a greater sensitivity and specificity $(95 \%$ and $90 \%$ respectively) than Doppler ultrasound for detecting extracranial vessel morphology, including carotid artery occlusions, stenosis, or dissections (75). As with MRI, it cannot be used in patients with pacemakers, metallic implants, and contraindications to MR contrast agents. Its use is limited in patients with severe claustrophobia. It also poses a significant risk of inducing 
nephrogenic interstitial fibrosis, secondary to use of gadolinium contrast agents in patients with renal dysfunction.

\section{Conclusion}

With the introduction of thrombolytic therapy in the treatment of acute ischemic stroke and a narrow therapeutic window (up to 4.5 hours from symptom onset in select patients), timely diagnosis is essential. CT imaging is required in the evaluation of acute stroke as it is quick and readily available. Several MRI modalities like DWI and ADC mapping are now being studied in the acute ischemic stroke diagnosis and management. Evaluation of cerebral vasculature in patients with ischemic stroke is important for endovascular therapy consideration. It is important to remember when considering an imaging modality that time is brain.

\section{Notes}

Author contributions: All authors have seen and approved the manuscript, and contributed significantly to the work.

Financial support: Authors declare that no financial assistance was taken from any source.

Potential conflicts of interest: Authors declare no conflicts of interest. Authors declare that they have no commercial or proprietary interest in any drug, device, or equipment mentioned in the submitted article.

\section{References:}

1. CDC, NCHS. Underlying Cause of Death 1999-2013 on CDC WONDER Online Database, released 2015. Data are from the Multiple Cause of Death Files, 1999-2013, as compiled from data provided by the 57 vital statistics jurisdictions through the Vital Statistics Cooperative Program. Accessed Feb. 3, 2015.

2. Mozaffarian D, Benjamin EJ, Go AS, et al. Heart disease and stroke statistics-2015 update: a report from the American Heart Association. Circulation. 2015 ;e29-322.

3. Sacco RL, Kasner SE, Broderick JP, et al. American Heart Association Stroke Council, Council on Cardiovascular Surgery and Anesthesia.; Council on Cardiovascular Radiology and Intervention.; Council on Cardiovascular and Stroke Nursing.; Council on Epidemiology and Prevention.;Council on Peripheral Vascular Disease.; Council on Nutrition, Physical Activity and Metabolism.. An updated definition of stroke for the 21st century: a statement for healthcare professionals from the American Heart Association/American Stroke Association. Stroke. 2013 Jul;44(7):2064-89.

4. González RG. Imaging-guided acute ischemic stroke therapy: From "time is brain" to "physiology is brain". AJNR Am J Neuroradiol. 2006 Apr;27(4):728-35.

5. Marshall RS. Progress in Intravenous Thrombolytic Therapy for Acute Stroke. JAMA Neurol. 2015 Aug;72(8):928-34. doi: 10.1001/jamaneurol.2015.0835.

6. Liebeskind DS. Imaging the future of stroke: I. Ischemia. Ann Neurol. 2009 Nov;66(5):574-90.

7. Liebeskind DS, Alexandrov AV. Advanced multimodal CT/MRI approaches to hyperacute stroke diagnosis, treatment, and monitoring. Ann N Y Acad Sci. 2012 Sep;1268:1-7.

8. Caplan LR, Wong KS, Gao S, Hennerici MG. Is hypoperfusion an important cause of strokes? If so, how? Cerebrovasc Dis. 2006;21(3):145-53. Review.

9. Ropper AH, Brown RH .Adams and victor's principles of neurology. 8th edition. New York: The McGraw Hill Companies, Inc; 2005, p662

10. Biller J, Love B.B, Schneck MJ. Vascular Diseases of the Nervous System : Ischemic Cerebrovascular Disease. In: Bradley WG, Daroff RB, Fenichel GM, Jankovic J, editors. Neurology in Clinical Practice. $5^{\text {th }}$ edition. Philadelphia. Elsevier; 2008, p1165-1166. 
11. Powers WJ, Raichle ME. Positron emission tomography and its application to the study of cerebrovascular disease in man. Stroke 1985;16(3):361-376.

12. Jones TH, Morawetz RB, Crowell RM, et al. Thresholds of focal cerebral ischemia in awake monkeys. J Neurosurg. 1981;54(6):773-82.

13. Powers WJ, Grubb RL, Jr, Darriet D, et al. Cerebral blood flow and cerebral metabolic rate of oxygen requirements for cerebral function and viability in humans. J Cereb Blood Flow Metab. 1985;5(4):600-8.

14. Astrup J, Siesjo BK, Symon L. Thresholds in cerebral ischemia - the ischemic penumbra. Stroke. 1981;12(6):723-5

15. Hossmann KA. Viability thresholds and the penumbra of focal ischemia. Ann Neurol. 1994;36(4):557-65.

16. Hossmann KA. Neuronal survival and revival during and after cerebral ischemia. Am J Emerg Med. 1983;1(2):191-7.

17. Copen WA, Schaefer PW, Wu O. MR perfusion imaging in acute ischemic stroke. Neuroimaging Clin N Am 2011;21(2):259-283

18. Ferrari $\mathrm{J}$ et al. Differences in process management and in-hospital delays in treatment with IV thrombolysis. PLoS One. 2013;8:e75378.

19. Hacke W, Kaste M, Bluhmki E, et al; ECASS Investigators. N Engl J Med. 2008;359:1317-1329

20. Demchuk AM, Hill MD, Barber PA, et al; NINDS rtPA Stroke Study Group, NIH. Importance of early ischemic computed tomography changes using ASPECTS in NINDS rtPA Stroke Study. Stroke. 2005;36:21102115.

21. Dzialowski I, Hill MD, Coutts SB, et al. Extent of early ischemic changes on computed tomography (CT) before thrombolysis: prognostic value of the Alberta Stroke Program Early CT Score in ECASS II. Stroke. 2006;37:973-978.

22. Tomura N, Uemura K, Inugami A, et al. Early CT finding in cerebral infarction: obscuration of the lentiform nucleus. Radiology. 1988;168(2):463-7

23. Truwit CL, Barkovich AJ, Gean-Marton A, et al. Loss of the insular ribbon: another early CT sign of acute middle cerebral artery infarction. Radiology 1990; 176:801.

24. von Kummer R, Holle R, Gizyska U, et al. Interobserver agreement in assessing early CT signs of middle cerebral artery infarction. AJNR Am J Neuroradiol. 1996;17:1743-1748.

25. Grotta JC, Chiu D, Lu M, et al. Agreement and variability in the interpretation of early CT changes in stroke patients qualifying for intravenous rtPA therapy. Stroke. 1999;30:1528-1533.

26. Schriger DL, Kalafut M, Starkman S, Krueger M, Saver JL. Cranial computed tomography interpretation in acute stroke: physician accuracy in determining eligibility for thrombolytic therapy. JAMA. 1998;279: 12931297.

27. Wardlaw JM, Dorman PJ, Lewis PC, Sandercock PA. Can stroke physicians and neurologists identify signs of early cerebral infarction on CT? J Neurol Neurosurg Psychiatry. 1999;67:651-653. 48.

28. Roberts HC, Dillon WP, Furlan AJ, et al. Computed tomographic findings in patients undergoing intra-arterial thrombolysis for acute ischemic stroke due to middle cerebral artery occlusion: results from the PROACT II trial. Stroke. 2002;33: 1557-1565.

29. von Kummer R, Nolte PN, Schnittger H, Thron A, Ringelstein EB. Detectability of cerebral hemisphere ischaemic infarcts by CT within $6 \mathrm{~h}$ of stroke. Neuroradiology. 1996; 38:31-33.

30. Barber PA, Demchuk AM, Zhang J, Buchan AM; ASPECTS (Alberta Stroke Programme Early CT Score) Study Group. Validity and reliability of a quantitative computed tomography score in predicting outcome of hyperacute stroke before thrombolytic therapy Lancet. 2000;355: 1670-1674. 52.

31. Demchuk AM, Coutts SB. Alberta Stroke Program Early CT Score in acute stroke triage (ASPECTS). Neuroimaging Clin N Am. 2005;15: 409-419, xii.

32. Barber PA, Demchuk AM, Hill MD, et al. The probability of middle cerebral artery MRA flow signal abnormality with quantified CT ischaemic change: targets for future therapeutic studies. J Neurol Neurosurg Psychiatry 2004, 75:1426-1430.

33. Kirchhof K, Welzel T, Mecke C, Zoubaa S, Sartor K: Differentiation of white, mixed, and red thrombi: value of CT in estimation of the prognosis of thrombolysis phantom study. Radiology 2003, 228:126-130.

34. Qureshi AI, Ezzeddine MA, Nasar A, et al. Is IV tissue plasminogen activator beneficial in patients with hyperdense artery sign? Neurology 2006, 66: 1171-1174.

35. Smith WS, Tsao JW, Billings ME, et al. Prognostic significance of angiographically confirmed large vessel intracranial occlusion in patients presenting with acute brain ischemia. Neurocrit Care. 2006;4:14-17.

36. Tomsick T, Brott T, Barsan W, et al. Prognostic value of the hyperdense middle cerebral artery sign and stroke scale score before ultraearly thrombolytic therapy. AJNR Am J Neuroradiol. 1996;17:79-85. 
37. Fiorelli M, Bastianello S, von Kummer R, et al. Hemorrhagic transformation within 36 hours of a cerebral infarct: relationships with early clinical deterioration and 3-month outcome in the European Cooperative Acute Stroke Study I (ECASS I)cohort. Stroke. 1999 Nov;30(11):2280-4.

38. Kidwell CS, Alger JR, Di Salle F, et al. Diffusion MRI in patients with transient ischemic attacks. Stroke. 1999;30:1174-1180

39. Tomandl BF, Klotz E, Handschu R, et al. Comprehensive imaging of ischemic stroke with multisection CT. Radiographics. 2003 May-Jun;23(3):565-92.

40. Torres-Mozqueda F, He J, Yeh IB, et al. An acute ischemic stroke classification instrument that includes CT or MR angiography: The Boston Acute Stroke Imaging Scale. AJNR Am J Neuroradiol. 2008;29:1111-7.

41. Lev MH, Farkas J, Rodriguez VR, et al. CT angiography in the rapid triage of patients with hyperacute stroke to intraarterial thrombolysis: accuracy in the detection of large vessel thrombus. J Comput Assist Tomogr. 2001;25:520-528.

42. Lev MH, Segal AZ, Farkas J, et al. Utility of perfusion-weighted CT imaging in acute middle cerebral artery stroke treated with intra-arterial thrombolysis: prediction of final infarct volume and clinical outcome. Stroke. 2001;32:2021-2028.

43. Hirai T, Korogi Y, Ono K, et al. Prospective evaluation of suspected stenoocclusive disease of the intracranial artery: combined MR angiography and CT angiography compared with digital subtraction angiography. AJNR Am J Neuroradiol. 2002;23:93-101.

44. Skutta B, Furst G, Eilers J, Ferbert A, Kuhn FP. Intracranial stenoocclusive disease: double-detector helical CT angiography versus digital subtraction angiography. AJNR Am J Neuroradiol. 1999;20:791-799.

45. Lubezky N, Fajer S, Barmeir E, Karmeli R. Duplex scanning and CT angiography in the diagnosis of carotid artery occlusion: a prospective study. Eur J Vasc Endovasc Surg. 1998;16:133-136

46. Srinivasan A, Goyal M, Al Azri F, Lum C. State of the art imaging of acute stroke. Radiographics. 2006;26:S75-S95.

47. Wintermark M, Flanders AE, Velthuis B, et al. Perfusion-CT assessment of infarct core and penumbra: receiver operating characteristic curve analysis in 130 patients suspected of acute hemispheric stroke. Stroke. 2006;37(4):979-85.

48. Eastwood JD, Lev MH, Provenzale JM. Perfusion CT with iodinated contrast material. AJR Am J Roentgenol. 2003;180(1):3-12.

49. Wintermark M, Maeder P, Thiran JP, et al. Quantitative assessment of regional cerebral blood flows by perfusion CT studies at low injection rates: a critical review of the underlying theoretical models. Eur Radiol. 2001;11(7):1220-30.

50. Wintermark M, Meuli R, Browaeys P, et al. Comparison of CT perfusion and angiography and MRI in selecting stroke patients for acute treatment. Neurology 2007;68:694

51. Koenig M, Kraus M, Theek C, et al. Quantitative assessment of the ischemic brain by means of perfusion related parameters derived from perfusion CT. Stroke 2001;32:431-7.

52. Wintermark M, Maeder P, Thiran JP, et al. Quantitative assessment of regional cerebral blood flows by perfusion CT studies at low injection rates: a critical review of the underlying theoretical models. Eur Radiol. 2001;11(7):1220-30

53. Wintermark M, Reichhart M, Thiran JP, et al. Prognostic accuracy of cerebral blood flow measurement by perfusion computed tomography, at the time of emergency room admission, in acute stroke patients. Ann Neurol. 2002;51(4):417-32.

54. Hellier KD, Hampton JL, Guadagno JV, et al. Perfusion CT helps decision making for thrombolysis when there is no clear time of onset. J Neurol Neurosurg Psychiatry 2006, 77:417-419.

55. Mohr JP, Biller J, Hilal SK, et al. Magnetic resonance versus computed tomographic imaging in acute stroke. Stroke. 1995;26(5):807-12.

56. Thomalla G, Rossbach P, Rosenkranz M, et al. Negative fluid-attenuated inversion recovery imaging identifies acute ischemic stroke at 3 hours or less. Ann Neurol. 2009;65(6):724-32.

57. Gonzalez RG, Schaefer P. In: Conventional MRI and MR Angiography of Stroke. Gonzalez RG, Hirsch JA, Koroshetz WJ, et al., editors. Springer; Berlin: 2006. pp. 115-37.

58. Fiebach JB, Schellinger PD, Gass A, et al. Stroke magnetic resonance imaging is accurate in hyperacute intracerebral hemorrhage: a multicenter study on the validity of stroke imaging. Stroke 2004;35(2):502-506

59. Fiehler J, Albers GW, Boulanger JM, et al. Bleeding risk analysis in stroke imaging before thromboLysis (BRASIL): pooled analysis of $\mathrm{T} 2 *$-weighted magnetic resonance imaging data from 570 patients. Stroke. 2007;38(10):2738-44. 
60. Barber PA, Darby DG, Desmond PM, et al. Identification of major ischemic change: diffusion-weighted imaging versus computed tomography. Stroke. 1999;30:2059-2065.

61. Fiebach JB, Schellinger PD, Jansen O, et al. CT and diffusion-weighted MR imaging in randomized order: diffusion-weighted imaging results in higher accuracy and lower interrater variability in the diagnosis of hyperacute ischemic stroke. Stroke. 2002;33:2206-2210.

62. González RG, Schaefer PW, Buonanno FS, et al. Diffusion-weighted MR imaging: diagnostic accuracy in patients imaged within 6 hours of stroke symptom onset. Radiology. 1999;210:155-162.

63. Lövblad KO, Laubach HJ, Baird AE, et al. Clinical experience with diffusion-weighted MR in patients with acute stroke. AJNR Am J Neuroradiol. 1998;19:1061-1066.

64. Lutsep HL, Albers GW, DeCrespigny A, et al. Clinical utility of diffusion-weighted magnetic resonance imaging in the assessment of ischemic stroke. Ann Neurol. 1997;41:574-580

65. Kunz A, Hahn G, Mucha D, et al. Echo-enhanced transcranial color-coded duplex sonography in the diagnosis of cerebrovascular events: a validation study. AJNR Am J Neuroradiol. 2006;27(10):2122-7.

66. Ozsunar Y, Sorensen AG. Diffusion- and perfusion-weighted magnetic resonance imaging in human acute ischemic stroke: technical considerations. Top Magn Reson Imaging 2000;11(5):259-272

67. Wu O, Schwamm LH, Sorensen AG. Imaging stroke patients with unclear onset times. Neuroimaging Clin N Am. 2011;21(2):327-344

68. Lansberg MG, Straka M, Kemp S, et al. MRI profile and response to endovascular reperfusion after stroke (DEFUSE 2): a prospective cohort study. Lancet Neurol 2012;11(10):860-867

69. Barber PA, Davis SM, Darby DG, et al. Absent middle cerebral artery flow predicts the presence and evolution of the ischemic penumbra. Neurology 1999, 52:1125-1132.

70. Sobesky J, Zaro Weber O, Lehnhardt FG, et al. Which time-topeak threshold best identifies penumbral flow? A comparison of perfusion-weighted magnetic resonance imaging and positron emission tomography in acute ischemic stroke. Stroke 2004, 35:2843-2847.

71. Grandin CB, Duprez TP, Smith AM, et al. Which MR-derived perfusion parameters are the best predictors of infarct growth in hyperacute stroke? Comparative study between relative and quantitative measurements. Radiology 2002, 223:361-370.

72. Gonzalez RG, Schaefer P. In: Conventional MRI and MR Angiography of Stroke. Gonzalez RG, Hirsch JA, Koroshetz WJ, et al., editors. Springer; Berlin: 2006. pp. 115-37.

73. Graves MJ: Magnetic resonance angiography. Br J Radiol 1997, 70:6-28.

74. Bash S, Villablanca JP, Jahan R, Duckwiler G, et al. Intracranial vascular stenosis and occlusive disease: evaluation with CT angiography, MR angiography, and digital subtraction angiography. AJNR Am J Neuroradiol. 2005;26:1012-1021.

75. Nederkoorn PJ, Elgersma OE, van der Graaf Y, et al. Carotid artery stenosis: accuracy of contrast-enhanced MR angiography for diagnosis. Radiology. 2003; 228: 677-682. 